\title{
Trichobezoar - A Case Report of Rapunzel Syndrome
}

\author{
S. Shanmuga Jayanthan ${ }^{1}$ \\ ${ }^{1}$ Consultant Radiologist, Department of Radiology and Imaging Sciences, Meenakshi hospital, Tanjore - 613005, Tamil Nadu, \\ India
}

Corresponding author: Dr. S. Shanmuga Jayanthan, 27, Third Cross Street, Subbaiya Pillai Nagar, Ammal Sathiram, Karaikal -609604, India

DOI: http://dx.doi.org/10.21276/ijcmsr.2019.4.3.36

How to cite this article: Shanmuga Jayanthan S. Trichobezoar - a case report of rapunzel syndrome. International Journal of Contemporary Medicine Surgery and Radiology. 2019;4(3):C168-C170.

\section{A B S T R A C T}

Introduction: Bezoars are ingested foreign materials present within the gastrointestinal system. They can be further classified based on their composition and trichobezoar is one subtype with hair as content. Rapunzel syndrome is a rare type of trichobezoar in which the hairball is seen extending into the small bowel. Delay in diagnosis may lead to serious complications like bowel obstruction and perforation.

Case report: A 10 year old female child presented with complaints of abdomen pain and generalised weakness for 4 months. On examination, the child had frontal alopecia and a palpable, painless, mobile mass was felt in the epigastrium. Since plain X-ray and ultrasound (USG) abdomen imaging findings were non-specific, further imaging with computed tomography (CT) was considered. Contrast enhanced CT (CECT) abdomen revealed well defined large floating heterodense non-enhancing mass with calcifications in the stomach, measuring $15 \times 6 \mathrm{~cm}$, with air bubbles retained in the interstices.

Conclusion: This case report highlights the importance of being aware of this rare condition and the role of various imaging modalities in the diagnosis as the delay in diagnosis may lead to complications. Although USG is the initial imaging modality, it is less specific in diagnosing bezoar. Hence, CECT should be the imaging modality of choice in all suspected cases.

Keywords: Trichobezoar, Rapunzel syndrome

\section{INTRODUCTION}

Bezoars are ingested foreign materials present within the gastrointestinal system. Based on their composition they are classified into phytobezoars, trichobezoars, lactobezoars and pharmacobezoars. Of which trichobezoars and phytobezoars are the more common forms. ${ }^{1}$ Rapunzel syndrome is a rare type of trichobezoar in which the hairball is seen extending into the small bowel. ${ }^{2}$ Clinical presentation may vary from milder degree of nausea, vomiting and epigastric discomfort to severe forms of small bowel obstruction and perforation. ${ }^{3}$ CECT is the ideal imaging modality of choice in the diagnosis of this condition, evaluating the extent and its complications. ${ }^{3}$

\section{CASE REPORT}

A rare diagnosis of Rapunzel syndrome was suspected in a 10 year old female child who presented with abdomen pain and generalised weakness. There was no history of psychiatric illness. Clinical examination revealed patchy areas of frontal alopecia (Figure 1) and a painless, mobile mass was felt in the epigastrium. Laboratory blood parameters showed reduced haemoglobin (Hb-8gm\%). Plain X-ray abdomen showed compact heterogeneous mottled mass with areas of calcification and crescent of air surrounding it (Figure 2). USG showed curvilinear hyperechoic area with posterior acoustic shadow in epigastrium (Figure 3). As the above imaging findings were non-specific, CECT was advised. CECT abdomen revealed a well-defined floating heterodense non-enhancing mass with calcifications in stomach, measuring $15 \times 6 \mathrm{~cm}$, with air bubbles retained in the interstices (Figure 4.a\&4.b). Another similar mass, measuring $3 \times 3 \mathrm{~cm}$, also noted in proximal small bowel loop (Figure 4.c). Upper GI endoscopy was further planned and showed a gastric trichobezoar with its tail extending into proximal duodenum (Figure 5.a \&5.b). Surgical treatment in the form of anterior gastrotomy was performed and the trichobezoar cast in the shape of stomach, measuring $25 \times 15$ $\mathrm{cm}$, was removed (Figure 6.a \& 6.b).

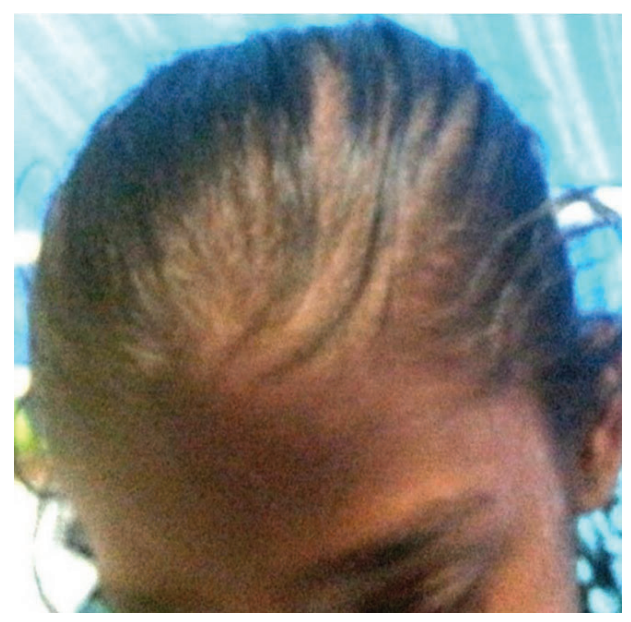

Figure-1: Frontal alopecia 


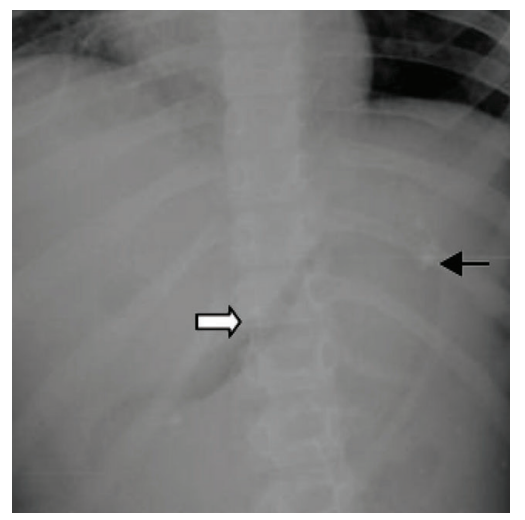

Figure-2: Plain X-ray abdomen shows mottled mass with calcification (black arrow) and crescent of air surrounding it (white arrow).

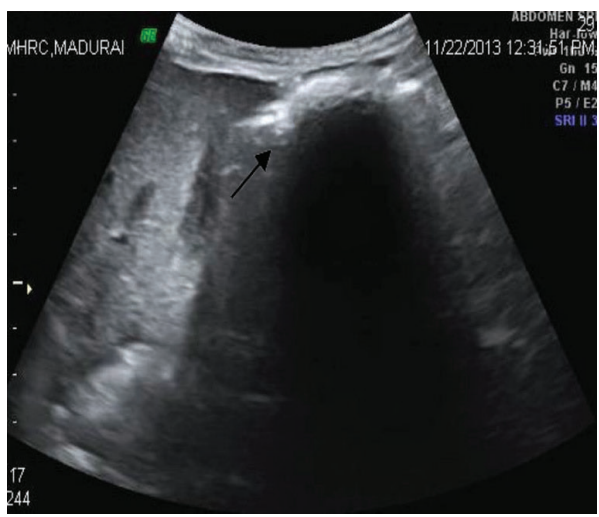

Figure-3: USG abdomen shows hyperechoic area with posterior acoustic shadow in the epigastrium (black arrow).
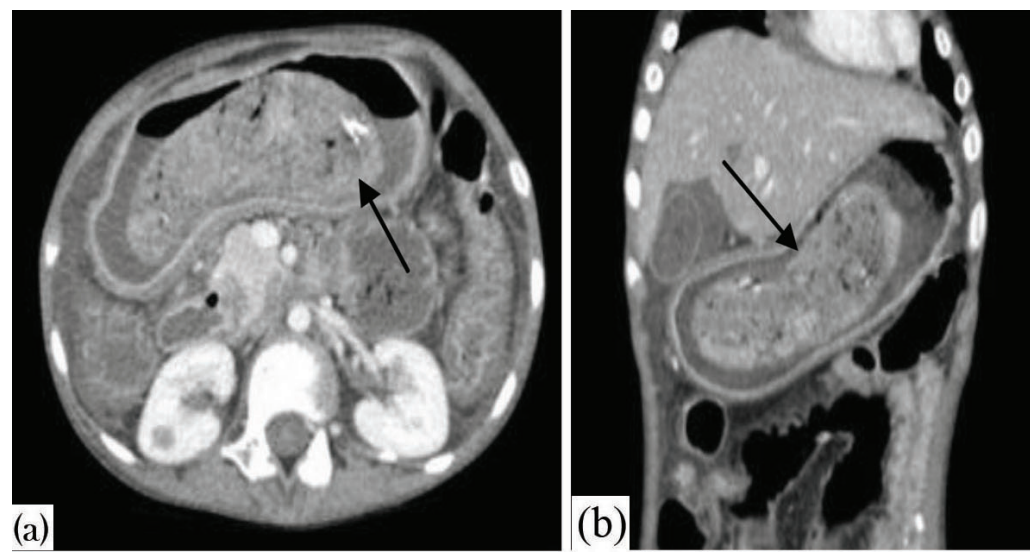

Figure-4: (a) Axial and (b) Coronal CECT abdomen show heterodense mass interspersed within it in the stomach; (c) Axial CECT abdomen shows simila
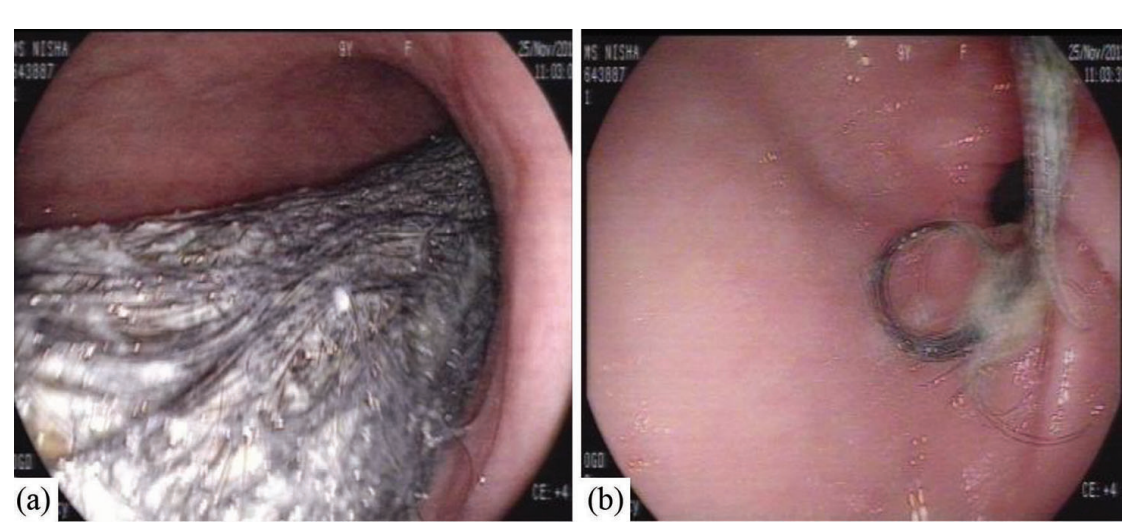

Figure-5 (a \& b): Shows the endoscopic appearance of gastric trichobezoar with its tail extending into proximal duodenum.
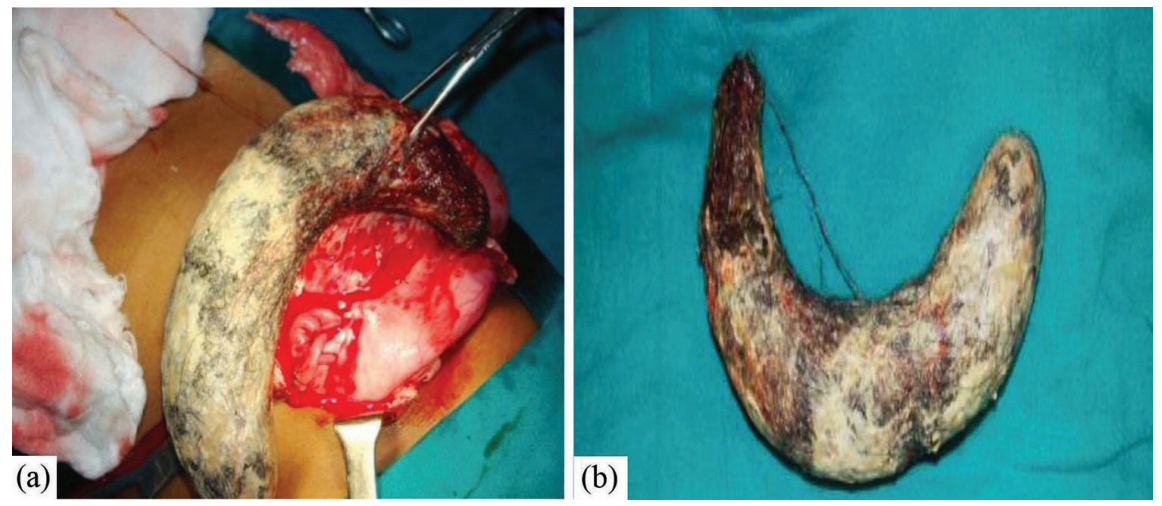

Figure-6 (a \& b): Shows the intraoperative pictures of trichobezoar. 


\section{DISCUSSION}

Bezoar is rare and uncommon diagnosis in clinical practice. It is more common in teenage females. ${ }^{4,5}$ Adding to the difficulty in diagnosis, history of trichophagia is present in only half of the cases. ${ }^{2}$ Clinical suspicion together with the help of radiological imaging, early diagnosis of this condition can be made. Though $\mathrm{x}$-ray and USG are the initial imaging modalities, they are often non-specific and inconclusive., CECT is the imaging modality of choice in patients with suspected clinical history and imaging findings. ${ }^{5}$ It can demonstrate bezoar as heterodense, non-enhancing, well defined, intraluminal content with mottled appearance. Besides diagnosis, the other advantages of CECT include precise demonstration of the extent of the bezoar, its complications like obstruction and perforation. ${ }^{5}$ Once the diagnosis is made, endoscopic removal of the content may be tried but most cases require surgical intervention in the form of laparoscopy, endoscopy or laparotomy depending on the size and location., ${ }^{3,5}$ Recurrence has been reported, especially in patients in whom no psychiatric treatment had been initiated ${ }^{4}$ and hence counselling should be offered in all such cases of Rapunzel syndrome.

\section{CONCLUSION}

The rarity and atypical presentation of this condition can lead to delay in diagnosis and complications. CECT is the imaging modality of choice in all suspected cases as it can not only diagnose the condition but also can demonstrate the extent and complications associated with it. Early treatment along with psychiatric counselling can help in better prognosis and lesser recurrence.

\section{REFERENCES}

1. Ripolles T, Garcia-Aguayo J, Martinez M-J, Gil P. Gastrointestinal bezoars: sonographic and CT characteristics. AJR Am J Roentgenol. 2001;177(1):6569.

2. Dhinakar M, Balkhair W. Rapunzel Syndrome: A Case Report. Oman Med J. 2010;25(4):e016.

3. Charan SPG, Annigeri VM, Kalavant AB, Simha PV and Halgeri AB. Jejunal Trichobezoar-A Rare Cause of Intestinal Obstruction in Children: A Case Report. SM J Pediatr Surg. 2018; 4(3): 1069

4. Czerwinska K, Bekiesinska-Figatowska M, Brzewski M, Gogolewski M, Wolski M. Trichobezoar, rapunzel syndrome, tricho-plaster bezoar - a report of three cases. Pol J Radiol. 2015;80(4):241-246.

5. Hamidi H, Muhammadi M, Saberi B, Sarwari MA. A rare clinical entity: huge trichobezoar. Int J Surg Case Rep. 2016;28(6):127-130.

Source of Support: Nil; Conflict of Interest: None

Submitted: 03-08-2019; Accepted: 19-08-2019; Published online: 04-09-2019 